\title{
The Producing functionals and its applications
}

\author{
Muhammadyusuf Yunusi \\ Tajik National University \\ Dushanbe, Tajikistan \\ yunusi@inbox.ru; www.yunusi.tj
}

\begin{abstract}
The report is devoted to one class so-called producing functionals and corresponding with its modeling generating spaces, some transformations building models equations and corresponding spaces are considered and investigated. It is shown that these transformations any point of Euclidean space $E^{m-1}$ (generating spaces $M^{m-1}$ ) transfer into some corresponding points $E^{m}$ (or $M^{m}$ ) and conversely. It is also proposed one class general equations which are described many processes of chancing and generating spaces. Besides it is shown that processes of generating spaces are determined by some no depending prescribes points. Given others applications and from economics and physics.
\end{abstract}

Index Terms - class so-called producing functionals, transformations, models equations

We shall consider the set

$$
\begin{gathered}
M_{n}^{s}=\left\{\alpha=\left(\alpha_{1}(t), \ldots, \alpha_{m}(t)\right):\right. \\
\left.\sum_{j=1}^{m} \alpha_{j}^{\frac{n}{n-s}}(t)=1,0 \leq \alpha_{j}(t) \leq 1, t \in T, j=1, m ; n>s\right\},
\end{gathered}
$$

where $m, n, s$ are natural, $n>s, s \geq 1, m \geq 2, T$ is a arbitrary set from $[0, \infty)$. Let $\alpha \in M=M_{n}^{s}$ and $\alpha, x \in$ $L_{m}^{n}(T)$ with norm $\|x\|=\left(\int_{T} \sum_{j=1}^{m}\left|x_{j}\right|^{n} d t\right)^{\frac{1}{n}}<\infty$. Let the set of producing functionals of type

$$
\mu(\alpha)=\left(\int_{T}\left(\sum_{j=1}^{m} \alpha_{j}\left|x_{j}\right|^{s}\right)^{\frac{n}{s}} d t\right)^{\frac{1}{n}}
$$

are given for all $\alpha \in M_{n}^{s}$ and $x \in L_{m}^{n}(T)$. The set of functional (1) with norm $\|\mu\|=\sup _{\alpha \in M_{n}^{s}} \mu(\alpha)$ is the normed space. It denote by $M$. Introducing the vector $y=K|x|$, where $K$ is the diagonal matrix with elements $\alpha_{j}^{1 / s}$ we have the space with norm $\mu(\alpha)=\|y\|_{L_{m}^{n, s}(T)}=\left(\int_{T}\left(\sum_{j=1}^{m}|y|^{s}\right)^{\frac{n}{s}} d t\right)^{\frac{1}{n}}$ . is also the normae space. It denote by ${ }_{M}^{T}(\alpha)$.

Theorem 1. At $\alpha=\alpha^{0} \in M_{n}^{s}$, where $\alpha_{j}^{0}=$ $\left(\frac{\left|x_{j}\right|^{n}}{\sum_{j=1}^{m}\left|x_{j}\right|^{n}}\right)^{\frac{n-s}{n}}, j=1, m$ the functional (1) has a maximal value $\|\mu\|=\left(\int_{T} \sum_{j=1}^{m}\left|x_{j}\right|^{n} d t\right)^{1 / n}=\|x\|_{L_{m}^{n}(T)},\|y\|_{L_{m}^{s, s}(T)} \leq$ $Z,\|y\|_{L_{m}^{n, s}(T)} \leq Z$ and what is more all maximal values of the functional (1) with different $x \in L_{m}^{n}(T)$ are solution of the equation $\sum_{j=1}^{m} X_{j}^{n}=Z^{n}$ where $X_{j}=\left(\int_{T}\left|x_{j}\right|^{n} d t\right)^{1 / n}, j=$ $1, \ldots, m ; Z=\|\mu\|$. Besides $M \in M(\alpha)$ for all $\alpha \in M_{n}^{s}$.

Proof. As

$$
\mu(\alpha)=\left(\int_{T}\left(\sum_{j=1}^{m} \alpha_{j}\left|x_{j}\right|^{s}\right)^{\frac{n}{s}} d t\right)^{\frac{1}{n}}=\left(\int _ { T } \left(\sum_{j=1}^{m-1} \alpha_{j}\left|x_{j}\right|^{s}+\right.\right.
$$$$
\left.\left.\left(1-\sum_{j=1}^{m-1} \alpha_{j}^{\frac{n}{n-s}}\left|x_{m}\right|^{s}\right)^{\frac{n-s}{n}}\right)^{\frac{n}{s}} d t\right)^{\frac{1}{n}} \text { and }
$$

$$
\begin{aligned}
& \mu(\alpha+\Delta \alpha)=\underset{T}{\left(\int _ { j = 1 } \left(\sum_{j=1}^{m-1}\left(\alpha_{j}+\Delta \alpha\right)\left|x_{j}\right|^{s}+\right.\right.} \\
& \left.\left.\left(1 \quad-\quad \sum_{j=1}^{m-1}\left(\alpha_{j}+\Delta \alpha\right)^{\frac{n}{n-s}}\left|x_{m}\right|^{s}\right)^{\frac{n-s}{n}}\right)^{\frac{n}{s}} d t\right)^{\frac{1}{n}} \\
& =\quad \int_{T}\left(\sum_{j=1}^{m-1} \alpha_{j}\left|x_{j}\right|^{s}+\left(1-\sum_{j=1}^{m-1} \alpha_{j}^{\frac{n}{n-s}}\left|x_{m}\right|^{s}\right)^{\frac{n-s}{n}}+\right. \\
& \sum_{j=1}^{m-1} \Delta \alpha_{j}\left|x_{j}\right|^{s}-\left(1 \quad-\quad \sum_{j=1}^{m-1} \alpha_{j}^{\frac{n}{n-s}}\left|x_{j}\right|^{s}\right)^{\frac{n-s}{n}-1} . \\
& \left(\frac{n-s}{n}\right)\left(\frac{n}{n-s} \sum_{j=1}^{m-1} \alpha_{j}^{\frac{n-s}{n}-1}\left|x_{m}\right|^{s} \Delta \alpha_{j}-\right. \\
& \left.\sum_{j=1}^{m-1} \alpha_{j}^{\frac{n}{n-s}-2}\left|x_{j}\right|^{s} \Delta \alpha_{j}^{2}\right)+\frac{1}{2}\left(\frac{n}{n-s}\right) \cdot\left(\frac{n-s}{n}\right) \cdot\left(\frac{n-s}{n}-1\right) . \\
& \left(1-\sum_{j}^{m-1} \alpha_{j}^{\frac{n}{n-s}}\left|x_{m}\right|^{s}\right)^{\frac{n-s}{n}-2} \cdot\left(\sum_{j=1}^{m-1} \alpha_{j}^{\frac{n}{n-s}}\left|x_{m}\right|^{s} \cdot \Delta \alpha_{j}\right)^{2}+ \\
& \left.\ldots+)^{\frac{n}{s}} d t\right)^{\frac{1}{n}} .
\end{aligned}
$$

Continuing this expansion from condition $\operatorname{grad} \mu(\alpha)=0$ we have next equation $\left|x_{j}\right|^{s}-$ $\left(1 \sum_{j=1}^{m-1} \alpha_{j}^{\frac{n}{n-s}}\right)^{-\frac{s}{n}} \cdot \alpha_{j}^{\frac{s}{n-s}} \cdot\left|x_{m}\right|^{s}=0$ for definition maximal points of the functional (1). As $\left|x_{j}\right|^{s}-\alpha_{m}^{-\frac{s}{n-s}} \cdot \alpha_{j}^{\frac{s}{n-s}} \cdot\left|x_{j}\right|^{s}=0$ and $\frac{\alpha_{j}^{\frac{s}{n-s}}}{\alpha_{m}^{\frac{s}{n-s}}}=\frac{\left|x_{j}\right|^{s}}{\left|x_{m}\right|^{s}}$ or $\frac{\alpha_{j}^{\frac{n}{n-s}}}{\alpha_{m}^{\frac{n}{n-s}}}=\frac{\left|x_{j}\right|^{n}}{\left|x_{m}\right|^{n}}$ after summation with regard to condition $\sum_{j=1}^{m} \alpha_{j}^{\frac{n}{n-s}}(t)=1$ we have $\alpha_{j}^{-\frac{n}{n-s}}=\frac{\sum_{j=1}^{m}\left|x_{j}\right|^{n}}{\left|x_{m}\right|^{n}}$. Hence $\alpha_{j}^{0}{ }^{\frac{n}{n-s}}=\frac{\left|x_{j}(t)\right|^{n}}{\sum_{j=1}^{m}\left|x_{j}(t)\right|^{n}}$. Such under $\alpha=\alpha^{0}$ the functional $\mu(\alpha)$ has a maximal value (using condition $\left.d^{2} \mu\right|_{\alpha^{0}}<0$ ). Besides at this point we have $\mu\left(\alpha^{0}\right)=\left(\int_{T}\left(\sum_{j=1}^{m} \alpha_{j}^{0}\left|x_{j}\right|^{s}\right)^{\frac{n}{s}} d t\right)^{\frac{1}{n}}=$ $\left(\int_{T}\left(\sum_{j=1}^{m}\left(\frac{\left|x_{j}(t)\right|^{n}}{\sum_{j=1}^{m}\left|x_{j}(t)\right|^{n}}\right)^{\frac{n-s}{n}}\left|x_{j}\right|^{s}\right)^{\frac{n}{s}} d t\right)^{\frac{1}{n}}$

$\left(\int_{T}^{T} \sum_{j=1}^{m}\left|x_{j}(t)\right|^{n} d t\right)^{\frac{1}{n}}=\|x\|_{L_{m}^{n}(T)}=\|\mu\|$. Introducing $X_{j}=\left(\int_{T}\left|x_{j}\right|^{n} d t\right)^{\frac{1}{n}}, j=1, \bar{m}, Z=\|\mu\|$ we have the equation $([1])$ :

$$
\sum_{j=1}^{m} X_{j}^{n}=Z^{n}
$$

It is to easy that $M \in M(\alpha)$ for all $\alpha \in A$.

Now we consider the model space of functionals $\hat{M}$ with norm

$$
\hat{\mu}(\alpha)=\left(\int_{T} \sum_{j=1}^{m}\left|x_{j}\right|^{n} d \beta_{j}(t)\right)^{\frac{1}{n}},
$$

where $x \in L_{m}^{n}(T), \beta_{j}(t)=\int_{0}^{t} \alpha_{j}(t) d t, \alpha \in \hat{A}=A_{s}^{n}$. Introducing notations $\hat{x}_{j}=\left(\int_{T}\left|x_{j}\right|^{n} d \beta_{j}(t)\right)^{\frac{1}{n}}, \hat{z}=\hat{\mu}(\alpha)$, we have equations

$$
\sum_{j=1}^{m} \hat{x}_{j}^{n}=\hat{z}^{n}
$$


The set of points $\left(\hat{x}_{1}, \ldots, \hat{x}_{m}\right)$ with norm $\hat{z}$ is formed the Euclidean model space $\hat{M}^{m}(\alpha), \alpha \in \hat{A}$. For functionals of type (3) from $\hat{M}$ also take place $\hat{M}=\hat{M}(\alpha)$.

Theorem 2. For any natural $n>1$ between to set of solutions (4) ( or (2) ) ( i.e. under $m=k-1$ and $m=k$ ) it take place next presentations:

$$
\begin{gathered}
\hat{x}_{j k}=\hat{x}_{12} \hat{x}_{j k-1}, \quad \hat{x}_{k k}=\hat{x}_{22} \hat{z}_{k-1}, \\
\hat{z}_{k}=\hat{z}_{2} \hat{z}_{k-1}, \quad j=1, k-1
\end{gathered}
$$

where $k=3,4, \ldots, m,\left(\hat{x}_{12}, \hat{x}_{22}\right)$ is some point of the special Plane with distance $\hat{z}_{2}=\left(\hat{x}_{12}^{n}+\hat{x}_{22}^{n}\right)^{\frac{1}{n}}$.

Proof. Let $\left(x_{1 k-1} \ldots, x_{k-1 k-1}, z_{k-1}\right)$ are solutions (4) under $m=k-1$ ( the sign of $\hat{.}$ is dropped ). We shall that $\left(x_{i k}, \ldots, x_{k k}, x_{k}\right)$ obtained by of (5) and are the solutions (4) under $m=k$. So that $\sum_{i=1}^{k-1} x_{i k-1}^{n}=z_{k-1}^{n}$, then multiplied both part of the last identify on $x_{12}^{n}$ we have: $x_{12}^{n} \sum x_{i k-1}^{n}=$ $x_{12}^{n} z_{k-1}^{n}$. From here $\sum_{i=1}^{k-1}\left(x_{12} x_{i k-1}\right)^{n}=\left(z_{2}^{n}-x_{22}^{n}\right) z_{k-1}^{n}$, and hence, using (5) we have: $\sum_{i=1}^{k} x_{i k}^{n}=z_{k}^{n}$, i.e equation (4) under $m=k$. Analogous, if $\left(x_{i k} \ldots, x_{k k}, z_{k}\right)$ also satisfied the equations (1), then it is easy to see $\left(x_{i k-1}, \ldots, x_{k-1 k-1}, z_{k-1}\right)$ also satisfied (4) under $m=k-1$.The transformation of (5) may be writhen in the form of type: $\hat{x}_{m}=\mu_{t} \hat{x}_{m-1}$, where $\hat{x}_{m}=\left(\hat{x}_{1 m-1}, \ldots, \hat{x}_{m-1 m-1}, \hat{z}_{m-1} \hat{z}_{m-1}\right), \hat{z}_{m-1}=$ $\left(\sum_{i=1}^{m-1} \hat{x}_{i m-1}^{1 / t}\right)^{t}$ and $\hat{x}_{m}=\left(\hat{x}_{1 m}, \ldots, \hat{x}_{m m}, \hat{z}_{m}\right), \hat{z}_{m}=$ $\left(\sum_{i=1}^{m} \hat{x}_{i m}^{1 / t}\right)^{t}, m=1,2 \ldots, \mu_{t}$ is diagonal matrix of $m+1$ th order

$$
\mu_{t}=\left(\begin{array}{cccccc}
\hat{x}_{12}^{t} & 0 & \ldots & 0 & 0 & 0 \\
0 & \hat{x}_{12}^{t} & \cdots & 0 & 0 & 0 \\
\vdots & \vdots & \ddots & \vdots & \vdots & \vdots \\
0 & 0 & \vdots & \hat{x}_{12}^{t} & 0 & 0 \\
0 & 0 & \vdots & 0 & \hat{x}_{22}^{t} & 0 \\
0 & 0 & \vdots & 0 & 0 & \hat{z}_{2}^{t}
\end{array}\right)
$$

where $\hat{x}_{12}, \hat{x}_{22}$ is some point of $\hat{M}^{2}$ with metric $\hat{z}_{2}=$ $\hat{x}_{12}+\hat{x}_{22}$ and $0<\hat{x}_{12}<e^{q}, 0<\hat{x}_{22}<e^{q}, 0<\hat{z}_{2}<2 e^{q}$ , and $0 \leq t \leq t_{k}, t_{k}<\infty, q=$ const $>0$. It is noticed that the transformation of $\mu_{t}$ transfers arbitrary point $\left(\hat{x}_{1 m-1}, \ldots, \hat{x}_{m-1 m-1}\right) \in \hat{M}^{m-1}$ with metric $\hat{z}_{m-1}$ and into some corresponding point $\left(\hat{x}_{1 m}, \ldots, \hat{x}_{m m}\right)$ from $\hat{M}^{m}$ with metric $\hat{z}_{m}$ for all $m \geq 3$ and has semi-group properties: $\mu_{t+s}=\mu_{t} \mu_{s}, 0 \leq t \leq t_{k}, 0 \leq s \leq t_{k}$ and its are linears, uniformly bounded and uniformly continued. Besides, the eignevalues of $\mu_{t}$ are represented in the form of: $\lambda_{j}=\hat{x}_{12}^{t}, j=$ $1, m-1 ; \lambda_{k}=\hat{x}_{22}^{t} ; \lambda_{k+1}=z_{2}^{t} ;\left\|\mu_{t}\right\|=\hat{z}_{2}^{t} ;\left\|\mu_{t}^{-1}\right\|<\infty$. The infinitesimal generating operator $K=\lim _{t \rightarrow 0} t^{-1}\left(\mu_{t}-I\right)$ is diagonal matrix and represented in the following way: $a_{i i}=\ln \hat{x}_{12}, i=1, \ldots, k-1, a_{k k}=\ln \hat{x}_{22}, a_{k+1 k+1}=\ln \hat{z}_{2}$, and what is more $R(m, K)=(q I-K)^{-1}, \mu_{t}=e^{t K}$, $K=\ln \mu_{t}^{1 / t}$. The transformation $\mu_{t}^{1 / t}$ is also transfered $\hat{M}^{k-1}$ into $\hat{M}^{k}$ under corresponding condition $\hat{x}_{12}^{1 / t}+\hat{x}_{22}^{1 / t}=$ $\hat{z}_{2}^{1 / t}, 0 \leq t \leq t_{k}, t_{k}<\infty$. The transformations $\mu_{t}, \mu_{t}^{-1}$ may be use to coding and decoding of corresponding input and output information. It is characterized in the following way:

$\hat{M}^{3}$
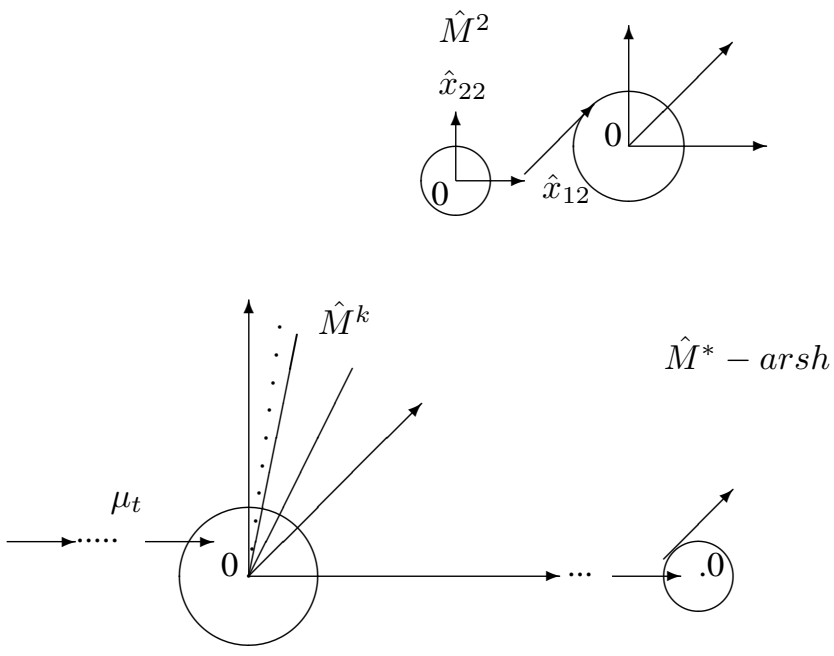

The plane of $\hat{M}^{2}$ is special plane and all points of the model spaces $\hat{M}^{2}(\alpha), \alpha \in M_{s}^{n}$ are depending from points of this plane. The plane of $\hat{M}^{2}$ is called prescribed set[1-3].

Theorem 3. Let the function $u=u\left(\hat{x}_{1}, \ldots, \hat{x}_{m}, \hat{z}\right)$, $\left(\hat{x}_{1}, \ldots, \hat{x}_{m}\right) \in \hat{M}^{m}, \hat{z}=\left(\sum_{j=1}^{m} \hat{x}_{j}^{n}\right)^{1 / n}$ is the density of some information flow ( some substance, or moving object, or so on ) and $L_{j}, j=1, m, L$ are some operators which are realizing changes of corresponding information flows then $\sum_{j=1}^{m}\left(L_{j} u\right)^{n}=(L u)^{n}$, are its general equations. Really, as $L u=\max _{\alpha \in \hat{A}} \sum_{i=1}^{m} \alpha_{i} L_{i} u$, Then at $\alpha_{i}=\alpha_{i}^{0}$ is corresponding value under which the right of part of the equation has the maximal value and this equation are equivalent. Besides this equation has solution if and only if when the predetermined system $L_{j} u=\phi_{j}, L u=\phi$, where $\phi_{j}=\phi_{j}\left(x_{1}, x_{2} \ldots x_{m}, z\right), j=1,2 \ldots m, \phi\left(x_{1}, x_{2}, \ldots, x_{m}, z\right)$ are solutions of the next functional equations has a solution $\sum_{j=1}^{m} \phi_{j}^{n}=\phi^{n}$, Having taken $\phi=Z_{m}=c_{m}, \phi_{j}=$ $X_{i m}=c_{i m}$ and using transformation (5) we have all "simple" solutions of the equations. For example let $c_{j}, c$ are some numbers solutions of equation $\sum_{j=1}^{m} c_{j}^{n}=c^{n}$ then $\phi_{j}=$ $c_{j} \phi\left(x_{1}, \ldots, x_{m}, z\right), \phi=c \phi\left(x_{1}, \ldots, x_{m}, z\right)$ for all $\phi()>$.0 and $\phi \in C$ are solutions of the equation (7). We may be also take $\phi_{j}()=.x_{j}^{2 / n}, \phi=c_{0} t^{2 / n}$ and considering process will be define in the set of $S_{0} \in S$, where $S_{0}=\left\{\left(x_{1}, \ldots, x_{m}, z\right)\right.$ : $\left.\sum_{j=1}^{m} x_{j}^{2}=c_{0}^{n} t^{2},-\infty<x_{j}<\infty, 0<t<\infty, n \geq 2\right\}$ where $z^{n}=c_{0}^{n} t^{2}$. The set $G_{0}$ is series orbit with radius $R=c_{0}^{n / 2} t$, $0<t<\infty$.In the case of $L_{j}=\frac{\partial^{k}}{\partial \hat{x}_{j}^{k}}, L=\frac{\partial^{k}}{\partial \hat{z}^{k}}, k \geq$ 1 general solutions of this equation is represented in the form of: $u\left(x_{1}, \ldots, x_{m}, z\right)=\varphi\left(x_{1}, \ldots, x_{m}, z\right)+\sum_{i=1}^{m} c_{i} \frac{x_{i}^{k}}{k !}+$ $\frac{c z^{k}}{k !},-\infty<x_{i}<\infty,-\infty<z<\infty$ or $u\left(x_{1}, \ldots, x_{m}, z\right)=$ $\varphi\left(x_{1}, \ldots, x_{m}, z\right)+\sum_{i=1}^{m} \frac{x_{i}^{k+2 / n}}{(1+2 / n) \ldots(k+2 / n)}+\frac{z^{k+2 / n}}{(1+2 / n) \ldots(k+2 / n)}$, and $\sum_{i=1}^{m} x_{i}^{2}=z^{2}, \quad-\infty<x_{i}<\infty, \quad-\infty<z<\infty$, where $c_{i}, c$ are the solutions of the equation (2) or (4), the function $\varphi($.$) is polyoma of the k-1$-th order. Coefficients 
of this polyoma we shall define with help of boundary and initial conditions. For example at $m=2, k=2$ if we are given $\left.\frac{\partial u}{\partial z}\right|_{z=0}=u_{1}\left(x_{1}, x_{2}\right),\left.u\right|_{z=0}=u_{0}\left(x_{1}, x_{2}\right)$ for definition of the function $\varphi($.$) we have: \varphi\left(x_{1}, x_{2}, z\right)=$ $u_{0}(0,0)+u_{1}(0,0) z+\frac{\partial u_{0}(0,0)}{\partial x_{1}} x_{1}+\frac{\partial u_{0}(0,0)}{\partial x_{2}} x_{2}+\frac{\partial u_{1}(0,0)}{\partial x_{1}} x_{1} z+$ $\frac{\partial u_{1}(0,0)}{\partial x_{2}} x_{2} z+\frac{\partial^{2} u_{0}(0,0)}{\partial x_{1} \partial x_{2}} x_{1} x_{2}+\frac{\partial^{2} u_{1}(0,0)}{\partial x_{1} \partial x_{2}} x_{1} x_{2} z$.

Definition of Numbers Tree. For any positive number, $N$ there are natural numbers $p, n, m>1$ and positive numbers $a_{i}, a_{i j}, \ldots, a_{i, m}$ for which take place $N^{p}=N_{m}^{p}, N_{m}^{p}=$ $a_{1}^{n}+a_{2}^{n}+\ldots+a_{m}^{n}, \quad a_{j}^{p}=a_{1 j}^{n}+a_{2 j}^{n}+\ldots+a_{m j}^{n}, \quad a_{i j}^{n}=$ $a_{1 i j}^{n}+a_{2 i j}^{n}+\ldots+a_{m i j}^{n}, \quad a_{i j k}^{p}=a_{1 i j k}^{n}+a_{2 i j k}^{n}+\ldots+a_{m i j k}^{n}$, $\ldots, a_{i j k \ldots s}^{p}=a_{1 i j k \ldots s}^{n}+a_{2 i j k \ldots s}^{n}$ and in last presentation members of the right part can not beat, are submitted as the final sum composed $n-t h$ degrees of some integers so-called by a basis of a tree[1]. 1). The number $N$ is uniquely represented as Numbers Tree representation: $N_{m}^{p}=$ $\sum k_{j_{q}} a_{i j j_{1} j_{2} \ldots j_{q}}^{n}$, where $k_{j_{q}}$ are numbers of occurrence of a basic element $a_{i i j_{1} \cdots j_{q}}$ in a tree of numbers. Representation for $N$ and corresponding Numbers Tree representation are optimum representation. More over with the help of transformation $a_{i m}=x a_{i m-1}, i=\overline{1, m-1}, \quad a_{m m}=y \sqrt[n]{N_{m-1}^{p}}, \quad N_{m}=$ $z N_{m-1}$, where $x^{n}+y^{n}=z^{p}$ we have the polynomial formulas $N_{m}^{p}=\left(x^{m-1}\right)^{n}+\sum_{i=2}^{m}\left(y x^{m-i} z^{\frac{p(i-2)}{n}}\right)^{n}$ or $N_{m}^{p}=X^{m-1}+Y \sum_{i=2}^{m} X^{m-i} Z^{(i-2)}$, and $N_{m}^{p}=X^{m-1}+$ $\sum_{i=2}^{m} A_{i} X^{m-i}$, . which describes the process of Numbers Tree grows and any complex object $\left(a_{i}, \ldots, a_{i m}\right), N_{m}$ is fully defined with the help of infinity or account numbers of elementary objects of type $(x, y, z)$, where $x^{n}+y^{n}=z^{p}$, $X+Y=Z, X=x^{n}, Y=y^{n}, Z=z^{p}, A_{\alpha_{1}}=Y Z_{\alpha_{2}}^{(i-2)}$.

Remark. Newton type Law $F=\gamma \frac{m_{1}^{\alpha_{1}} m_{2}^{\alpha_{2}} \ldots m_{k}^{\alpha_{k}}}{R^{k}}$ (in particular $F=\frac{\gamma}{R^{2}}\left(\frac{m}{m_{0}}\right)^{\alpha_{1}}\left(\frac{E}{E_{0}}\right)^{\alpha_{2}}$ ), i.e. $\frac{F R^{k}}{\gamma}=m_{1} \alpha_{1} \ldots m_{k}{ }^{\alpha_{k}}$, and $\ln \frac{F R^{k}}{\gamma}=\alpha_{1} \ln m_{1}+\ldots+\alpha_{k} \ln m_{k}$ take the form $f^{n}=\left(\ln m_{1}\right)^{n}+\ldots+\left(\ln _{n} m_{k}\right)^{n}$ after maximization last function on the set $\sum_{i=1}^{k} \alpha_{i}^{\frac{n}{n-1}}=1$, where $f=\max \ln \frac{F R^{k}}{\gamma}$ . More over Newton type Law and Einstein of type equation $E^{n}=\left(m c^{2}\right)^{n}+(P c)^{n}$ on base our representation has similar algebraic representation of next type $Z^{n}=\sum^{k} X_{i=1}^{n}$. And from here we received polynomial formulas $(2)$. More over from here we have that for Neuston law $X_{1}=\ln m_{1}, \ldots$, $X_{k}=\ln m_{k}, Z=f$ and for Einstein Equation we have where $x=m c^{2}, y=P c, z=E, p=2, n=2$.

\section{REFERENCES}

[1] M.Yunusi. The Method of Extreme Representations and its applications. - Dushanbe,TNU, 2020, - 52p.

[2] M.Yunusi. Information tekhnology ...-Dushanbe,2019, - 340p.

[3] M.Yunusi. Model of Numbers Tree and its applications,International Congress Mathemations,The Book Abstracts, ICM 2010, Hyderabad, India, 2010, pp.531-532. 